\title{
Ueber den Zustand des Goldes im Goldquarz.
}

Vorläufige Mitteilung ').

Von P. P. von Weimarn (St. Petersburg).

(Eingeg. am 9. Dezember 1912)

\section{Allgemeines.}

Unter den die Bildung von goldhaltigen Quarzadern erklärenden Hypothesen existiert auch eine, die auf der Annahme basiert, daB es kieselsaures Gold gibt, welches sich elwas in einem einen Ueherschut von Kieselsäure enthaltenden Wasser löst. Eine derartige Lösung steigt in den Spalten, die Kieselsäure scheidet sich, da bei Druck- und Temperaturabnahme sich auch die Löslichkeit verringert, in Form von Quarz aus, das kieselsaure Gold fällt ebenfalls aus und geht bei der Reduktion in metallisches Gold über. Es ist möglich, daB auf diese Weise (R. Brauns, Chemische Mineralogie [St. Petersburg 1904], 357-358) dic goldhaltigen Quarzadern entstanden sind. Als Bestätigung dieser Hypothese (vgl. Brauns I. c.) dient teilweise das Vorkommen von Gold in den Inkrustationen der Kieselquellen.

Auf die Frage von der Existenz des kieselsauren Goldes und seiner Rolle bei der Bildung von goldhaltigem Quarz kam ich beim Betrachten von einigen Mustern goldhaltigen Quarzes, die mir 1910 der Assistent unzeres Probier - Laboratoriums Berg-Ingenieur Dr. A. A. Ssementschenko zeigte. Ich wunderte mich besonders über die Muster von milchigweibem, durchscheinendem Quarz, die trotz eines merklichen Gehaltes an Gold äuBerlich dasselbe nicht erkennen lieben. Augenscheinlich befindet sich das Gold in solch einem Quarz in einem überultramikroskopischen Zustande. Der überultramikroskopische dispersoide Zustand des Goldes im Quarz konnte durch die grobe Schutzwirkung der Kieselsăure hervorgerufen sein. lch brachte diese Wirkung in Zusammenhang mit intermediärem Vorhandensein von kiesel-

1) Diese vorlăufige Mitteilung ist durch die in Koll.Zeitschr. 10, 265 (1911) erschienene Abhan Jlung von E. Hatschek und A. L. Sim on veranlast. Die Bearbeitung des Problems über den Goldzustand im Quarzgestein wurdc von mir vor zwei Jahren begonnen, aber von cinem andern Standpunkt aus, als der, den E. Hats chek und A. L. Sim on einehmen. Inwicweit mein Standpunkt richtig ist, kann erst nach Beendigung meiner Untersuchungen entschieden werden, dic hinsichtlich des experimentellen Teils sehr viel Zeit erfordern. Diese Mitteilung soll nur den experimentellen Weg anzeigen, den ich zur Losung des erwahnten Problems bis jetzt eingeschlagen habe und auch weiterhin verfolge. saurem Golde und unternahm im August 1910 eine systematische experimentelle Untersuchung in" dieser Richtung.

2. Ueberdie Schutzwirkung derKieselsäure gegenüber dem dispersoiden Golde nach den Daten der früheren Untersuchungen.

Gemä6 den Untersuchungen von $R$. $Z$ sig mon $\mathrm{dy}^{2}$ ) ubt das Kieselsäuresol keine Schutzwirkung gegenüber dem dispersoiden Golde aus. F. Küs per $\mathbf{t}^{3}$ ) erhielt stabile dispersoide Ag- und Au-Lösungen, indem er als Schutzdispersoid eine Lösung von kieselsaurem Natron verwandte, er schreibi: "Wenn man bedenkt, daB bei der Umsetzung der Elektrolyte Natriumnitrat entsteht, so folgt aus dieser Beständigkeit, daB die Schutzwirkung des Wasserglases - nach R. Zsigmondy gegenüber dem roten Goldkolloid gleich Null - hier recht ausgiebig ist $\left.{ }^{4}\right)$." Ich glaube, daB in den Versuchen von $F$. K ü s pert die Stabilität der dispersoiden $\mathrm{Ag}$ - und $\mathrm{Au}$ Lösungen dadurch hervorgerufen wurde, daB durch Formalin die dispersoiden Teilchen der Gold- und Silbersilikate reduziert wurden; unter diesen Bedingungen verbanden sich natürlich die dispersoiden Gold- und Silberteilchen mit den bei der Reduktion der Silikate entstehenden Kieselsäureteilchen. Eine derartige Anschauung findet weiterhin einige Bestätigung

3. Ueber die spontane Reduktion von Gold beim Zusammenmischen von

Goldchloridchlornatrium-und Natriumsilikat-Lösungen.

Vorläufige Versuche mit konzentrierten Goldchlorid-und Natriumsilikat-Losungen haben gezeigt, dak, wenn auch kieselsaures Gold als eine stabile stöchiometrische chemische Verbindung existiert, so doch nur unter besonderen Bedingungen, denn bei Reaktionen in den Aequivalenten nahen Mengen erhält man Gallerten, welche im Laufe der Zeit spontan metallisches Gold von verschiedenem Dispersitätsgrade aus147

) R.Zsigmondy, Kolloidchemie (Leipzig 1912),

3) The Svedberg, Die Methoden zur Herstellung kolloider Lobsungen (Dresden 1909), 35.

4) L. C. 79 
scheiden. Um den Mechanismus der Reduktion des Goldes unter den erwähnten Bedingungen näher kennen zu lernen und um die die Stabilität des kieselsauren Goldes erhöhenden faktoren zu finden, beschloß ich, systematisch den Einflub der Konzentration der in der Einleitung erwähnten reagierenden Lösungen und den Ueberschub von Natriumsilikat auf den Verlauf des Prozesses zu studieren. Zu diesem Zweck wurde am 27. August 1910 der erste Versuch in dieser Richtung angestellt. Fürs erste ist es mir gelungen, den Prozeb der spontanen Reduktion des Goldes bei der Reaktion

$$
\mathrm{Na}_{2} \mathrm{SiO}_{3}+\mathrm{AuCl}_{3} \cdot \mathrm{NaCl}
$$

beiLösungskonzentrationen zwischen 0,1-1 Proz. zu erforschen, wobei von den reagierenden Lösungen gleiche Volumina zusammengegossen wurden. Bei den angeführten Grenzwerten der Konzentrationen erfolgt keine Ausscheidung der Kieselsäure und keine Gelatinierungserscheinungen der Lösung, aber eine allmähliche Bildung von kolloidem Golde. Beim Zusammengieben einer Goldsalz- und einer NatriumsilikatLösung verschwindet die gelbe Färbung der ersteren Lösung im Laufe von einigen Minuten und die Flüssigkeit erscheint dem unbewaffneten Auge vollkommen wasserklar. Nimmt man 0,1 prozentige Lösungen, so bleibt die Flüssigkeit einige Tage hindurch farblos, nimmt allmählich eine rosae, eine lilae Färbung an und geht schließlich in Blau über. Die Niederschlagsausscheidung beginnt ungefähr nach einem Jahr, die Koagulation verläuft dagegen sehr langsam und die Lösung bleibt noch lange gefärbt. Wenn die Verdampfung nicht verhindert wird, so erscheint der Niederschlag auch an der freien Flüssigkeitsoberfläche; er ist anfänglich dunkelblau, später schwarz, getrocknet gewöhnlich golden.

Bej nahen 0,1 prozentigen Konzentrationen erscheint die Fürbung der Lösung bedeutend schneller rosa, wird immer schwerer wahrgenommen und geht schnell in lila über. Wenn die Koagulation bei großen Konzentrationen auch schneller eintritt als bei kleinen, so sind bis zur vollen Entfärbung der Lösungen doch zirka $1 \frac{1}{2}$ Jahre erforderlich. Die erwähnten Erscheinungen erkläre ich durch die Entstehung des instabilen Goldsilikats und dessen Selbstreduktion. Aller Wahrscheinlichkeit nach wirkt auf die Selbstreduktion das Licht beschleunigend ein und das Goldsilikat wird nur dann stabil sein, wenn auf das Goldquantum eine verhăltnismäBig sehr groBe Menge Kieselsäure kommt. Die Versuche in dieser Richtung werden von mir ebenfalls noch ausgeführt.
4. Ueber die Struktur des Goldniederschlages.

Mikro- uud ultramikroskopisch habe ich von Zeit zu Zeit zweierlei Goldniederschläge untersucht: der erste ist ein beim Zusammengieben (am 27. August 1910) von 0,1 prozentigen Lösungen erhaltener Niederschlag; der zweite durch $\mathrm{Zu}_{\mathrm{u}}$ sammengieben (am 9. Oktober 1910) von 1 prozentigen Lösungen entstanden. Dem We sen

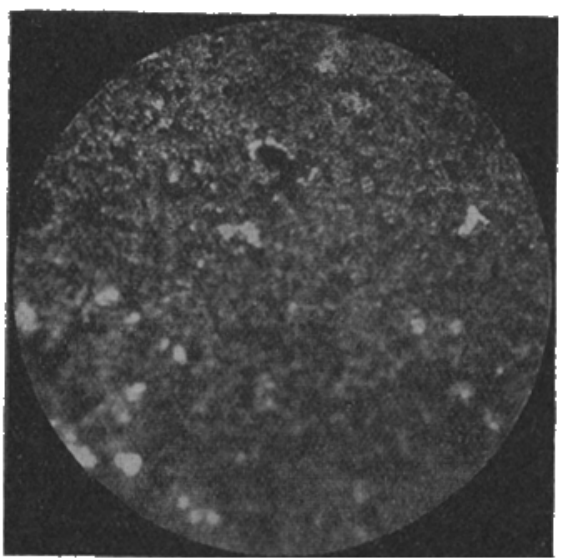

Fig. 1

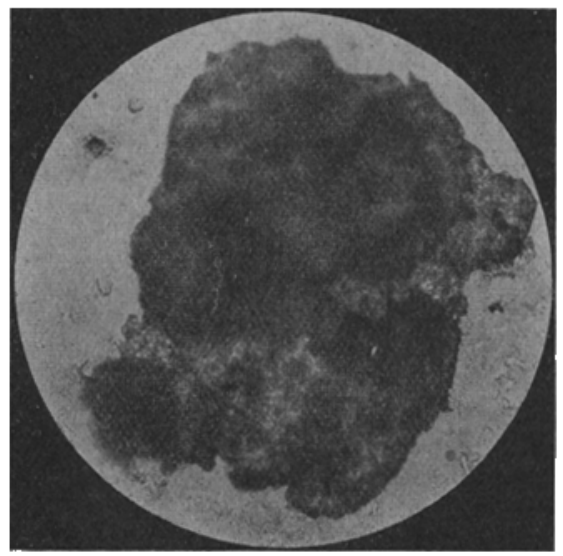

Fig. 2

nach habe ich $z$ wischen den beiden Niederschlagen keinen Unterschied gefunden. Im ersten Stadium sind die Niederschläge hochdispers, so daB es unmöglich ist, die Form der denselben bildenden Körner zu bestimmen. Ungefähr $11 / 2$ Jahre besteht der Niederschlag zum Teil aus Bruchstïcken von Dendriten (Fig. 1) und kleinen Goldstücken von unregelmäBiger form. Das in Figur 2 abgebildete Goldstückchen erinnert sehr an das im Quarz vorkommende gediegene Gold. 
KegelmäBige holuedrische Goldkristalle habe ich nicht benhachten können und erkläre das damit. daB bei dem hăufigen Herausnehmen von Niederschlagsproben die Flüssigkeit aufgerührt und dadurch die Kristallformen im Anfangsstadium zerstort wurden ${ }^{5}$ ). Es muB bemerkt werden, dak man auch ohne mikroskopische Untersuchung von dem Vorhandensein eines gewöhnlichen kristallinischen metallische॥ Goldniederschlages überzeugt sein kann, dafür spricht schon die typische goldgelbe Farbe des getrockneten Niederschlages. Interessant ist es; daB nach den Beobachtungen von $L$ iversidg $\mathrm{e}^{6}$ ) das Gold im Quarz nur in Ausuahmefällen deutlich kristallinisch auftritt, gewöhnlich findet es sich in Form von unregelmäBigen kleinen Schuppen, Körnern und Faden; deutlich kristallinisch kommt es in Hohlräumen oder in weicheren Mitteln, wie z. B. Kalkspat, Ton oder Serpentinstein vor.

5. Einige allgemeine Bemerkungen über die Kristallisation aus dispersoiden Lösungen.

Beim langsamen ZusammengieBen von verdünnten Gold- und Silbersalzlösungen mit den Lösungen von verschiedenen Reduktionsmitteln haben schon lange verschiedene Forscher deutliche Kristalle von diesen Metallen erhalten (R. Brauns 1. c. 261) ${ }^{7}$ ). Das Ethaltell von Kristallen in den eben erwahnten Fallen dient von dem Standpunkt, an den ich mich bei meinen dispersoidchemischen Untersuchungen gehalten habe, als ein Beweis auch für die Kristallinität des dispersoiden Goldes, weil eine je de Kristallisation mit dem dispersoiden $\mathrm{Zu}$ stande beginnt, obgleich in vielen Fallen die Dauer des dispersoiden Stadiums so gering ist (oder die Partikelchen so klein), dab dasselbe sich der Beobachtung entzieht ${ }^{8}$ ). Aber selbst in den Fällen, wo das dispersoide Stadium von längerer Dauer ist, kann man bei einiger Geduld die Entstehung ler deutlichen Kristalle verfolgen. Beim kolloiden Silber habe ich vor einigen Jahren dasselbe bestätigt gefunden ${ }^{3}$. Bezüglich der Kristallisation des kolloiden Sil-

8) Vgl. P.P. von We I ma rn; Koll.-Zeitschr. 7, 258 (1910) Wahmehmung von $M$. Frankenteim).

9) Angefuhrt im Mineralogiekursus von Prof. Dr. G. G. Le bedew (russ. Ausgabe, St. Petersburg 1907), 3 .

$7)$ Vgl. ebenfalls die in meiner Abhandlung in der Koll.-Zeitschr. 7, 258 (1910) angefürten Beobachtungen von M. L. Frankenheim.

9. P. von W eimarn, Grundzige d. Dispersoidchemie (Dresden 1911), 35 und ff.

9) Deutsch z. B. Koll.-Zeitschr. 4, 317; 5, $62 \mathrm{ff}$. bers möchte ich zur Vermeidung von Mibverständnissen einige Benierkungen machen. Schon Carey $L \in a^{10}$ ) hat die Entstehung von sonderbaren Kristallen, Nadeln und Prismen (!) beobachtet, die durch Wasserzusatz zerfallen und zu einer grünen glanzenden Masse trocknen. H. A m b r o $n^{11}$ ) erhielt ebenfalls vielleicht noch sonderbarere äuBerst feine Kristalle, welche denselben Pleochroismus besaBen, wie die silberhaltige Gelatine und welche zuweilen bei der Aenderung der Begrenzungsflächen in isotrope Kristalle übergehen. Da das gew öhnliche Silber nicht in Prismen kristallisiert, nicht quillt und nicht die Fähigkeit besitzt. plötzlich seine Begrenzungsflächen zu ändern und in irgendwelche neue ,isotrope" Kristalle zu zerfallen, so kann man die Kristalle von Carey Lea und $H$. Ambronn nicht für gew $8 \mathrm{hnliche}$ Kristalle des gew öhnlichen Silbers ansehen. Deshalb halte ich es für wichtig zu betonen, dab ich bej lingerem Stehen des Silbersols die allergewöhnlichsten Kristalle des gewöhnlichen Silbers erhalten habe, und zwar auBer den charakteristischen Dendriten auch noch holoedrische Mikrokristalle ${ }^{\text {2) }}$.

Das erwähnte Erhalten von Kristallen (Bruchstücke von Dendriten) auch wieder des gew 0 hnlich en Goldes von "goldgelber" Farbe dient als Bestatigung des von mir vielfach ausgesprochenen Grundsatzes von der Allgemeinheit der Kristallisationserscheinungen. Auf diese Weise erbält man aus den typischen kolloiden Lösungen, wie z. B. $\mathrm{Al}(\mathrm{OH})_{3}, \mathrm{Au}, \mathrm{Ag}, \mathrm{Kri-}$ stalle $\left.{ }^{13}\right)$. Wichtig ist es, daB in allen derartigen Fallen als Hauptgrund für die Kristallbildung nicht die Umkristallisation ${ }^{14}$ ) anzusehen ist, sondern das unmittelbare Wachstum auf Kosten der überultramikroskopischen Partikelchen und die Vereinigung der benachbarten dispersen Teilchen zu groberen unter dem Einfluß von Kristalllsationskräften. Der ProzeB der Entstehung von Mikrokristallen muBte bei der Umkristallisation (Verzehren der kleinen Teilchen durch die gröberen) unvergleichlich langsamer veriauten.

6. Das Erhalten eines dem Kassiuspurpur analogen dispersen Systems mit Kieselsaure.

Bei einem Versuch mit den ang efubrten(1Proz.) $\mathrm{Na}_{2} \mathrm{SiO}_{3}$ - und $\mathrm{Au} \mathrm{Cl}_{3} \cdot \mathrm{NaCl}-\mathrm{Lo}$ : ingen balve ich

19) 12) Zitiert nach R. Zsigmondy, 1. c. 18L. 12) R. Zsigmondy, 1. c. 132 (1912), berichtet, dab auch er ubniliches beobachtet hat.

19) Vgl. meine A handlung. Zur Lehre von den Zuständen der Materie: in der Köll-Zeltschr.

14) Koll. Zeltschr. 3, $289-290$ (1906); 5,63 (1909). 
in einem Trockenschrank über $\mathrm{H}_{2} \mathrm{SO}_{4}$ einen Teil des (farblos erscheinenden) Filtrats vom Niederschlage des kolloiden Goldes bis zur Trockne eingedämpft; hierbei bedeckte sich der Boden des Gefäbes mit einer stellenweise pur" purrot gefärbten Kieselsäureschicht, d. h es entstand ein sowohl dem Kassiuspurpur als auch dem von von mir vor mehreren Jahren aus dispersoidem Silber und Aluminiumhydroxyd erhaltenes disperses analoges System ${ }^{15}$ ). Die ultramikroskopische Untersuchung solcher purpurroter Flecke zeigte, daß wir es hierbei mit einem dispersen System $z$ tun haben, in dem das dispersoide Gold mit den Kieselsäureteilchen so innig vermengt ist, dat es nicht gelingt, dasselbe als getrennte Ultramikronen wahrzunehmen.

\section{Ueber die Entstehung des Goldes in den $Q u a r z a d e r n$.}

lch bin weit entfernt, auf Grund der angeführten experimentellen Daten eines noch durchaus nicht zu Ende geführten Studiums die Genesis der goldhaltigen Quarzadern zu untersuchen, nehme aber an, dall die Daten vollkommen genügen, um die Se lbstreduktion

13) Koll.-Zeitschr. 5, 66 (1909). des Goldes in Lösungen zu behaupten, welche Kieselsäure oder deren Salze in einem gelösten Zustande oder überhaupt in einem hochdispersen Zustande enthalten. Meine Versuche zeigen, daA die Anwesenheit von Reduktionsmitteln nicht $u n b e d i n g t ~ n o t w e n d i g$ ist zur Entstchung von metallischem Golde unter den Versuchsbedingungen von $F$. $K$ üspert und teilweise E. Hatschek und Simon, d. h. in einem Dispersionsmittel, welches Kieselsäure und ihre Salze in hochdispersem Zustande enthält. Damit will ich natürlich nicht behaupten, daB bei der Mannigfaltigkcit der Entstehung von Gold in der Natur nicht diese oder andere Prozesse der Reduktion von diesen oder jenen Goldsaizen stattfinden können. Ich neige $z$ der Annahme, daB bei der Entstehung von goldhaltigen Quarzadern eine wesentliche ( $\mathrm{mog}$ licherweise eine dominierende) Rolle die gleichzeitige Ausscheidung von Quarzkristallen und kieselsaurem Golde aus einem Kieselsäure enthaltenden Dispersionsmittel gespielt hat. Es ist sehr wahrscheinlich, dab hierbei sowohl kieselsaures Gold als auch überultramikroskopisches Gold in den Quarzkristallen eingeschlossen wurde. Die weiteren Versuche werden zeigen, inwieweit eine derartige Annahme berechtigt ist.

\section{Ueber die theoretische Möglichkeit einer Chromo-Ultramikroskopie.}

Von Wolfgang Ostwald (Leipzig).

\section{I.}

Der Verfasser hat wiederholt darauf hingewiesen ${ }^{1}$, daß bei der Deutung undifferenzicter sog. a mikrosk opischer Ultrabilder sehr häufig der Fehler gemacht wird, aus dem Vorhandensein eines undifferenzierten Uitrabildes ohne weiteres auf cinen außerordentlich hoh en Dispersitätsgrad zu schließen. Dies ist in der Tat mehrfach zu Unrecht geschehen und hat $z$. B. gelegentlich zu dem völlig irrtümlichen Schluß geführt, daß solvatisierte Emulsoide im allgemeinen höher disperse Systeme seien als Suspensoide. Auf $\mathrm{e}$ ine Fehlerquelle dieses Schlusses ist bereits schon von $H$. Siedentopf und $R, Z$ sigmondy, von $C h r$. Fü $c h t b a$ ue $\left.r^{2}\right)$ u. a. hingewiesen worden; dies ist eine zu dichte Lagerung der Teilchen. Wie der letztgenannte Forscher

1) Siehe z. B. Koll.-Zeitschr. 11, 251 (1912).

2) Chr. Füchtbauer, Zeltschr. f. physik. Chem. 48, 552 (1904). sehr richtig hervorhebt, kann z. B. aus dem undifferenzierten Lichtkegel mancher kritischer Flüssigkeitsgemische nicht ohne weiteres geschlossen werden, daß die Dimensionen der Teilchen unterhalb der ultramikroskopischen Sichtbarkeitsgrenze liegen, sondern nur, daB sie anscheinend enger zusammenliegen als etwa 0,1 Lichtwellenlänge usw. Ein zweiter Grund - - und dies ist der in vorliegender Mitteilung besonders in Betracht gezogene kann nun darin liegen, daB die Brechungsdifferenzen zwischen disperser Phase und Dispersionsmittel $z \mathbf{u} k$ le in sein, als daB Licht in merklicher Menge abgebeugt werden könnte, trotzdem die Teilchen durchaus übermikroskopische physische Dimensionen besitzen. Eine Amikroskopie kann also einzig durch diesen optischen Mangel entstehen, ganz analog dem Fall vom groben Glaspulver in einem genau gleichstark brechenden Medium, welches auch makro-, mikro- wie ultramikroskopisch unsichtbar bleiben mub. 